\title{
Anastomotic reinforcement with omentoplasty reduces anastomotic leakage for minimally invasive esophagectomy with cervical anastomosis
}

This article was published in the following Dove Press journal:

Cancer Management and Research

\author{
Dong Zhou \\ Quan-Xing Liu \\ Xu-Feng Deng \\ Hong Zheng \\ Xiao Lu \\ Ji-Gang Dai \\ Li Jiang
}

Department of Thoracic Surgery, Xinqiao Hospital, Third Military

Medical University, Chongqing,

People's Republic of China
Correspondence: Li Jiang; Ji-Gang Dai Xinqiao Hospital, Third Military Medical University, Chongqing 400037, People's Republic of China

Tel/fax + 862368774724

Email jiangli780830@gmail.com;

691057831@qq.com
Purpose: Anastomotic leakage is the most feared postoperative complication after esophagectomy. Omentoplasty, wrapping the omentum around the alimentary tract anastomosis, is thought to decrease the anastomotic leakage rate. The purpose of this clinical study is to investigate the use of omentoplasty to reinforce cervical esophagogastrostomy after minimally invasive esophagectomy (MIE).

Patients and methods: In this retrospective study, the data of 160 consecutive patients who underwent cervical esophagogastrostomy after MIE between September 2012 and May 2015 were analyzed, 87 who underwent omentoplasty (group A) and 73 who did not undergo omentoplasty (group B). The primary outcome was the incidence of anastomotic leakage and anastomotic strictures after the operation. Secondary outcomes were other complications and mortality rate. Univariate and multivariate analysis of variables associated with an increased risk for anastomotic leak was performed.

Results: The median age was 61 years (range, 37-82 years). The anastomotic leakage rates were $4.6 \%(4 / 87)$ in group $A$ and $15.1 \%(11 / 73)$ in group B $(P=0.023)$. There was no statistical significance in anastomotic stricture rates between group $\mathrm{A}(6.9 \%)$ and group $\mathrm{B}(9.6 \% ; P=0.535)$. No difference was noted in other complications between the groups. There was a trend toward lower leak-associated mortality rates for group A $(0 \%)$ compared with that for group B $(4.1 \%)$. Conclusion: Cervical esophagogastrostomy with omentoplasty is more effective than esophagogastrostomy without omentoplasty for the prevention of anastomotic leakage in MIE with cervical anastomosis. Omentoplasty could be used as an adjunct technique to reduce the incidence of anastomotic leakage in cervical esophagogastrostomy following MIE.

Keywords: omentoplasty, cervical esophagogastrostomy, minimally invasive esophagectomy, anastomosis leakage, stricture

\section{Introduction}

Esophageal cancer is the sixth leading cause of death due to cancer and the eighth most common cancer in the world. According to official statistics from China, there were approximately 478,000 newly diagnosed esophageal cancer patients and 375,000 associated deaths in 2015. ${ }^{1}$ The overall 5-year survival rate for this cancer is dismal, no better than $20 \%$. $^{2}$ Anastomotic leakage in particular is an important early postoperative complication having considerable impact on morbidity, mortality, and quality of life. ${ }^{3}$

Various surgical techniques are used for esophagectomy to produce better outcomes. Minimally invasive esophagectomy (MIE) was introduced in the 1990s for esophageal cancer and gained increasing popularity, with the aim of decreasing overall morbidity and mortality related to esophagectomy. ${ }^{4,5}$ In contrast to this reasoning, however, in a 
recent meta-analysis by Zhou et $\mathrm{al}^{6}$ in which 5,537 patients in 43 studies were reviewed, MIE was not associated with decreases in incidence of anastomotic leakage or stricture rate after esophagectomy for esophageal cancer, and therefore, there was no decrease in the morbidity and mortality related to the procedure.

Omentoplasty in esophagogastric anastomosis has been reported to decrease the incidence of postoperative anastomotic leakage, but its utility has not been evaluated in cervical esophagogastrostomy following MIE. ${ }^{7-9}$ Therefore, the aim of this study is to evaluate anastomotic leakage, strictures, and other complications in patients who underwent cervical esophagogastrostomy following MIE with or without omentoplasty.

\section{Patients and methods}

\section{Patients}

This study protocol was approved by the ethics committee of the Third Military Medical University, Chongqing, People's Republic of China, and written informed consent was obtained from all the patients before surgical intervention. Between September 2012 and May 2015, 738 patients with esophageal cancer were operated on at our institute. Among these, patients who did not undergo cervical esophagogastrostomy with MIE, underwent neoadjuvant therapy, or withheld consent for the omentoplasty technique were excluded from the study.

\section{Surgery}

At our institution, during the study period, all patients underwent McKeown MIE. After induction of general anesthesia and insertion of a double-lumen endotracheal tube with plans for deflation of the right lung and isolated left lung ventilation, the patient was placed in the left lateral decubitus position. Four ports were placed: in the 7 th intercostal space at the midaxillary line, in the 4th intercostal space at the anterior axillary line, in the 8th intercostal space at the scapular line, and in the 10th intercostal space at the midaxillary line.

After completion of the thoracoscopic esophagectomy with nodal dissection in the left lateral decubitus position, the patient was turned to the supine position. The abdominal section was performed laparoscopically. Four ports were placed: one-third of the distance from the umbilicus to the xiphisternum and in the left upper, left lateral, and right lateral quadrants.

The majority of the stomach was fashioned into a gastric tube using a liner stapler (CDH Ethicon; Johnson \& Johnson, New Brunswick, NJ, USA), and the procedure was repeated two or three times. Approximately $10-15 \mathrm{~cm}$ of the pedicled omentum was mobilized, with 6-8 $\mathrm{cm}$ remaining attached to the top of the gastric tube close to the proposed line of gastric resection (Figure 1).

After cervical lymph node dissection, the gastric tube, together with the pedicled omentum, was routinely delivered through the posterior mediastinum and up to the cervical area (Figure 2). For esophagogastric anastomosis, the cervical esophagus was exposed on the left side of the neck, and the esophagogastrostomy was performed $\sim 2 \mathrm{~cm}$ distal to the end of the gastric tube using the staple technique. The omentoplasty, wrapping the pedicled omentum around the esophagogastric anastomosis, was performed in the omentoplasty group (Figure 3). All the patients were transferred to

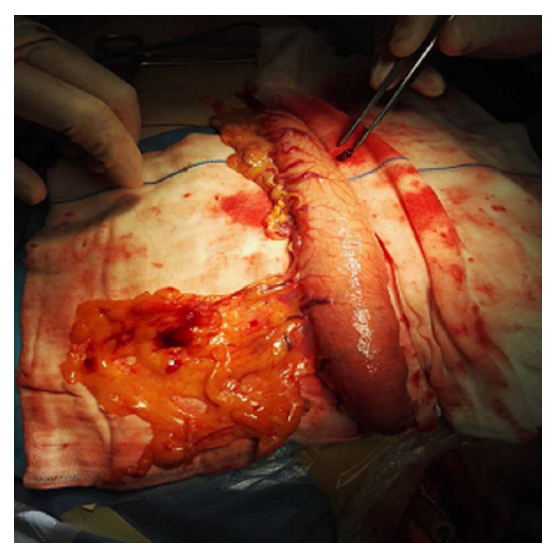

Figure I Pedicled omentum.

Note: A gastric tube was made using a linear stapler with $6-8 \mathrm{~cm}$ of the pedicled omentum, with 6-8 cm remaining of which was attached to the top of the gastric tube, close to the proposed line of the gastric resection.

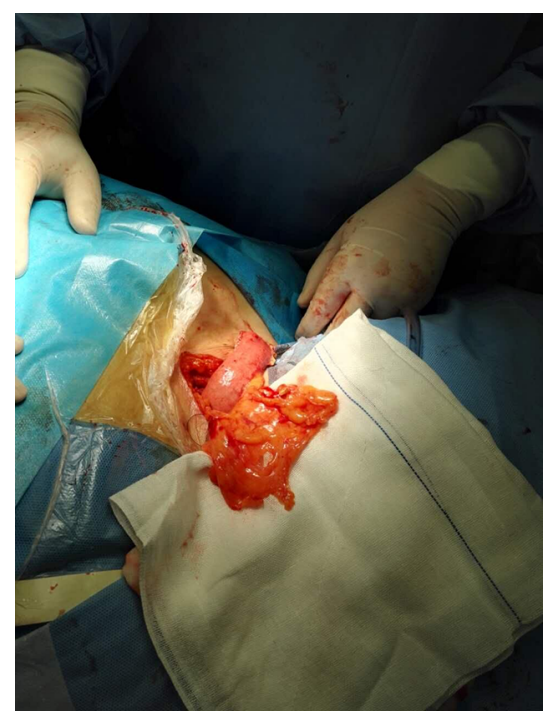

Figure 2 Pedicled omentum around the esophagogastric anastomotic site after esophagectomy.

Note: The gastric tube, together with the pedicled omentum, was delivered to the cervical area. 
the intensive care unit for 48-72 h. A group of four surgeons performed all surgical procedures during this study.

\section{Follow-up}

The primary outcomes were the rate of anastomotic leakage and the rate of benign anastomotic stricture in group A and group B. The diagnosis of anastomotic leakage and stricture was done by clinical or radiological evidence. Patients who could not eat, had symptoms of obstruction during swallowing, or had an anastomotic diameter less than $1 \mathrm{~cm}$ during endoscopy were diagnosed as having an anastomotic stricture. Patients in whom food residue leaked from the neck wound after eating or who were found to have leakage at endoscopy were diagnosed as having anastomotic leakage. All patients were followed up by the operating surgeon monthly for 2 years postoperatively. The median follow-up of surviving patients was 19 months (range, 4-39 months).

\section{Statistical analyses}

The $\chi^{2}$ test and Fisher's exact test were used for the qualitative data. Comparisons between groups were done using the independent samples $t$-test for independent samples in case of normal data distribution and the Mann-Whitney $U$ test in case of non-normal data distribution. Multivariable model used for prediction of anastomotic leakage was logistic regression. Odds ratios summarized the association between predictors and the presence of anastomotic leakage. $P<0.05$ was regarded as statistically significant. All statistical analyses were performed with SPSS statistical software package 13.0 (SPSS Inc., Chicago, IL, USA).

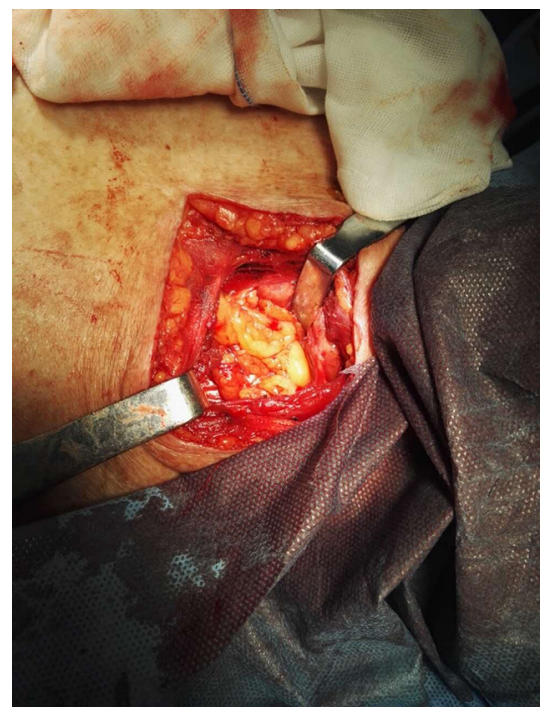

Figure 3 Cervical area anastomosis.

Note: The esophagogastric anastomosis was completed using the staple technique in the cervical area.

\section{Results \\ Characteristics of patients}

The clinical characteristics of the patients are shown in Table 1. In all, 165 consecutive patients diagnosed with esophagus cancer underwent total MIE with cervical anastomosis using the McKeown approach. Of whom, 160 were followed up until death or the end of the study period. The follow-up rate was $97 \%$. There were 133 men and 27 women with a mean age of 61 years (range, 37-82 years). The median operation time was 295 minutes (range, 174-676 minutes). The tumor was located in the upper thoracic esophagus in 41 patients, middle thoracic esophagus in 80 patients, and lower thoracic esophagus in 39 patients.

The pathologic characteristics of the patients are shown in Table 1 . The majority of patients had squamous cell carcinoma (78.1\%). The patients were staged using the tumor, node, metastasis classification of malignant tumors according to the American Joint Committee on Cancer; 25 patients had Stage I, 61 had Stage II, 62 had Stage III, and 12 had Stage IV

Table I Patient characteristics

\begin{tabular}{|c|c|c|c|}
\hline Characteristics & $\begin{array}{l}\text { Omentoplasty } \\
(\mathrm{n}=87)\end{array}$ & $\begin{array}{l}\text { No } \\
\text { omentoplasty } \\
(n=73)\end{array}$ & $P$ value \\
\hline \multicolumn{4}{|l|}{ Age (years) } \\
\hline Median & 61.5 & 64.2 & 0.725 \\
\hline Range & $37-81$ & $42-82$ & \\
\hline \multicolumn{4}{|c|}{ Length of stay (days) } \\
\hline Median & 9.6 & 10.5 & 0.637 \\
\hline Range & $6-21$ & $6-24$ & \\
\hline \multicolumn{4}{|l|}{ Sex, n (\%) } \\
\hline Male & 7I (82) & $62(85)$ & 0.576 \\
\hline Female & $16(18)$ & $11(15)$ & \\
\hline \multicolumn{4}{|l|}{ Location, n (\%) } \\
\hline Upper & $23(26)$ & $18(25)$ & 0.698 \\
\hline Middle & $45(52)$ & $35(48)$ & \\
\hline Lower & $19(22)$ & $20(27)$ & \\
\hline \multicolumn{4}{|l|}{ Stage, n (\%) } \\
\hline 1 & $15(17)$ & $10(14)$ & 0.342 \\
\hline II & $35(40)$ & $26(36)$ & \\
\hline III & $30(35)$ & $32(44)$ & \\
\hline IV & $7(8)$ & $5(6)$ & \\
\hline \multicolumn{4}{|l|}{ Tumor depth, n (\%) } \\
\hline TI & $17(20)$ & $13(18)$ & 0.780 \\
\hline $\mathrm{T} 2$ & $39(44)$ & $37(5 \mathrm{I})$ & \\
\hline T3 & $31(36)$ & $23(3 I)$ & \\
\hline \multicolumn{4}{|l|}{ Histology, n (\%) } \\
\hline $\begin{array}{l}\text { Squamous cell } \\
\text { carcinoma }\end{array}$ & $67(77)$ & $58(79)$ & 0.710 \\
\hline Other & $20(23)$ & $15(2 \mid)$ & \\
\hline \multicolumn{4}{|c|}{ Lymph nodes harvested } \\
\hline Median & 11.8 & 12.6 & 0.821 \\
\hline Range & $2-23$ & $3-21$ & \\
\hline
\end{tabular}


disease. Most patients had T2 tumors $(n=76)$, whereas 30 patients had T1 tumors and 54 patients had T3 tumors. Of note, the median number of lymph nodes harvested was 12 (range, 2-23).

\section{Primary outcomes}

Anastomotic leakage occurred in a total of $9.4 \%$ of all included patients. A significantly smaller number of anastomotic leakages occurred after McKeown MIE with omentoplasty than without omentoplasty (4.6\% [4/87] versus $15.1 \%$ [11/73], respectively, $P=0.023$ ). In group $\mathrm{A}$, one patient was clinically diagnosed and three patients were radiologically diagnosed; in group B, seven patients were clinically diagnosed and two patients were radiologically diagnosed $(P=0.491)$.

Malignant anastomotic stricture was not observed in either group during the first 6-month follow-up period. There were no statistical differences in the postoperative benign anastomotic stricture rate between those treated with and without omentoplasty (6.9\% [6/87] versus 9.6\% [7/73], respectively, $P=0.535)$. All strictures occurred within 1 year after the operation, and the medium duration from operation to the development of benign stricture was 4.8 months in group A and 3.6 months in group B (Table 2).

\section{Other postoperative complications}

Postoperatively, we observed no significant difference in pulmonary $(P=0.536)$, pneumonia $(P=0.874)$, cardiovascular $(P=0.743)$, or wound complication $(P=0.676)$ or hospital readmission $(P=0.913)$ rates between group A and group B. However, we observed that omentoplasty did decrease leakage-associated mortality in MIE with cervical anastomosis. None of the patients in group A and three patients in group B died of anastomotic leakage (Table 3).

\section{Preoperative variables associated with anastomotic leakage}

Preoperative patient variables and the univariate association with anastomotic leakage are listed in Table 4. Of these variables, renal insufficiency and diabetes were associated with a statistically significant increase in the risk for anastomotic leakage. Smoking, alcohol abuse, pulmonary insufficiency, cardiac insufficiency, and hypertension did not increase the risk for anastomotic leakage (Table 4). We have performed a logistic regression analysis with the impact of omentoplasty, renal insufficiency, and diabetes on postoperative leakage rates. Multivariable analysis of preoperative factors including renal insufficiency and diabetes identified to be statistically associated with an increased risk for anastomotic leakage. Omentoplasty constitutes an independent factor that decreases the risk for anastomotic leakage by the multivariable analysis (Table 5).

\section{Discussion}

To our knowledge, this is the largest study analyzing the influence of omentoplasty on cervical esophagogastric anastomotic leakage rate in MIE. Our data demonstrated a significantly decreased anastomotic leakage rate when the anastomosis was reinforced with an omental pedicle flap. Anastomotic leakage developed in two patients in group A $(4.6 \%)$ and nine patients in group B (15.1\%), and the leakages occurred on postoperative days $5-12$, which is similar to those reported in the literature.

Table 2 Anastomotic stricture and leakage in two groups

\begin{tabular}{llll}
\hline Variable & Omentoplasty $(\mathbf{n}=\mathbf{8 7})$ & No omentoplasty $(\mathbf{n}=\mathbf{7 3})$ & $\boldsymbol{P}$ value \\
\hline Anastomotic leak, $\mathrm{n}(\%)$ & $4(4.6)$ & $\mathrm{II}(\mathrm{I} 5 . \mathrm{I})$ & 0.023 \\
$\quad$ Clinical & $3(3.5)$ & $9(12.3)$ & $0.49 \mathrm{I}$ \\
$\quad$ Radiologic & $\mathrm{I}(\mathrm{I.1})$ & $2(2.7)$ & $7(9.6)$ \\
Anastomotic stricture, $\mathrm{n}(\%)$ & $6(6.9)$ & 3.6 & 0.535 \\
$\quad$ Time to development of stricture (months) & 4.8 & 0.324 \\
\hline
\end{tabular}

Table 3 Other postoperative complications and mortality in two groups

\begin{tabular}{llll}
\hline Variable & Omentoplasty $(\mathbf{n}=\mathbf{8 7}), \mathbf{n}(\%)$ & No omentoplasty $(\mathbf{n}=\mathbf{7 3}), \mathbf{n}(\%)$ & $\mathbf{P}$ value \\
\hline Pneumothorax & $3(3.5)$ & $5(6.8)$ & 0.536 \\
Pneumonia & $9(10.0)$ & $7(9.5)$ & 0.874 \\
Wound infection & $6(6.9)$ & $3(4.1)$ & 0.676 \\
Cardiovascular complications & $11(12.6)$ & $8(11.0)$ & 0.743 \\
Hospital readmission & $5(5.7)$ & $3(4.1)$ & 0.913 \\
Leak-associated mortality & $0(0)$ & $3(4.1)$ & 0.093 \\
\hline
\end{tabular}


Table 4 Univariate analysis of variables and association with anastomotic leak

\begin{tabular}{|c|c|c|c|}
\hline Characteristics & $\begin{array}{l}\text { Leakage } \\
(\mathrm{n}=15), \mathrm{n}(\%)\end{array}$ & $\begin{array}{l}\text { No leakage } \\
(n=145), n(\%)\end{array}$ & $P$ value \\
\hline Smoking & & & 0.496 \\
\hline $0=\mathrm{No}$ & $7(46.7)$ & 81 (55.9) & \\
\hline $\mathrm{I}=\mathrm{Yes}$ & $8(53.3)$ & $64(44.1)$ & \\
\hline Alcohol abuse & & & 0.595 \\
\hline $0=\mathrm{No}$ & $10(66.7)$ & $106(73.1)$ & \\
\hline $\mathrm{I}=$ Yes & $5(33.3)$ & 39 (26.9) & \\
\hline Pulmonary insufficiency & & & 0.901 \\
\hline $0=\mathrm{No}$ & $12(80.0)$ & II4 (78.6) & \\
\hline $\mathrm{I}=$ Yes & $3(20.0)$ & $31(2 \mid .4)$ & \\
\hline Cardiac insufficiency & & & 0.408 \\
\hline $0=\mathrm{No}$ & $9(60.0)$ & $102(70.3)$ & \\
\hline $\mathrm{I}=$ Yes & $6(40.0)$ & $43(29.7)$ & \\
\hline Renal insufficiency & & & 0.024 \\
\hline $0=\mathrm{No}$ & II (73.3) & I33 (9|.7) & \\
\hline $\mathrm{I}=$ Yes & $4(26.7)$ & $12(8.3)$ & \\
\hline Diabetes & & & 0.027 \\
\hline $0=$ No & $9(60.0)$ & I 30 (89.7) & \\
\hline $\mathrm{I}=\mathrm{Yes}$ & $6(40.0)$ & $24(10.3)$ & \\
\hline Hypertension & & & 0.120 \\
\hline $0=\mathrm{No}$ & $10(66.7)$ & II 4 (78.6) & \\
\hline $\mathrm{I}=$ Yes & $5(33.3)$ & $31(2 I .4)$ & \\
\hline Omentoplasty & & & 0.023 \\
\hline $0=\mathrm{No}$ & II (73.3) & $62(42.8)$ & \\
\hline $\mathrm{I}=$ Yes & $4(26.7)$ & $83(57.2)$ & \\
\hline
\end{tabular}

Table 5 Multivariate analysis of variables and association with anastomotic leak

\begin{tabular}{llll}
\hline Characteristics & OR & $\mathbf{9 5 \% ~ C l}$ & $\boldsymbol{P}$ value \\
\hline Renal insufficiency & 2.333 & $1.62 I-4.126$ & 0.040 \\
Diabetes & 3.418 & $1.815-5.367$ & 0.020 \\
Omentoplasty & 0.717 & $0.532-0.901$ & 0.040 \\
\hline
\end{tabular}

Abbreviation: $\mathrm{OR}$, odds ratio.

Although we found that omentoplasty did decrease the anastomotic leakage rates in MIE with cervical anastomosis, our study did not demonstrate a significant difference in anastomotic stricture occurrence between patients who underwent omentoplasty and those who did not. Anastomotic stricture developed in six patients in group A (6.9\%) and occurred in seven patients in group B $(9.6 \%)$.

Surprisingly, we found that omentoplasty did decrease leakage-associated mortality in MIE with cervical anastomosis. None of the patients in group A and three in group B died of anastomotic leakage. All three patients died due to severe lung infection caused by the inflow of liquid into the chest. When patients who underwent omentoplasty developed anastomotic leakage, it was limited by the omentum, and the gastric fluid or food residue was successfully drained from the neck incision. Furthermore, several sutures were placed to maintain the position of the omentum between the stomach and the esophagus to ensure an anastomosis without tension.

A recent study using a Japanese nationwide web-based database in which 5,354 esophageal cancer patients were reviewed showed that the frequency of leakages remains high, with an incidence rate of $13.3 \% .{ }^{10}$ Moreover, cervical anastomosis carries a higher risk for anastomotic leakage than intrathoracic anastomosis. As shown in the study by van Workum et al, ${ }^{11}$ the risk was less than $10 \%$ for intrathoracic anastomosis compared with $10 \%-25 \%$ for cervical anastomosis. Due to the high mortality and morbidity associated with anastomotic leakage, several authors have argued for the use of prophylactic interventions to reduce its impact and/or incidence. Therefore, there are numerous recommendations to avoid this condition, including wrapping the stomach around the anastomosis, stapling devices, tunnel esophagogastrostomy, triangulating stapling technique, and wrapping an omental graft around the anastomosis. ${ }^{12-16}$

Some authors have suggested covering the esophagogastric anastomosis with the pedicled omental flap, which was reported to have the advantage of reducing the occurrence of leakages. ${ }^{17-22}$ Bhat et $\mathrm{al}^{7}$ adopted this technique for creation of esophagogastric anastomosis, which was the first prospective randomized controlled trial to evaluate the influence of omentoplasty on esophagectomy. In total, 97 of 194 patients with esophageal cancer underwent esophagogastrostomy with wrapping of the pedicled omentum around the esophagogastric anastomosis. Of whom, $14(14.43 \%)$ patients in the group without omentoplasty experienced anastomotic leakage, but it was only seen in three (3.09\%) patients in the group with omentoplasty $(P=0.005)$. The authors recommended omental pedicle transposition to decrease the anastomotic leakage rate. Similarly, in 2011, Dai and coworkers ${ }^{8}$ conducted the second randomized trial, including 255 patients, to evaluate the influence of omentoplasty. Of whom, 127 patients received anastomotic omental reinforcement. The anastomotic leakage rate was $1 \%$ in the group with omentoplasty versus $6 \%$ in the group without omentoplasty $(P=0.05)$. The results showed that neither the rate nor the severity of anastomotic failure decreased in patients who underwent omentoplasty.

Although multiple studies have demonstrated the successful use of endoscopic stenting for the management of anastomotic leakage, its utility has not been reported in cervical esophagogastrostomy following MIE. Our clinical experience has proven that anastomotic leakage and leakageassociated mortality can be markedly reduced with the help of a pedicled omental graft technique. One possible reason 
is that the pedicled omentum acts as a source of granulation tissue, increases collateral blood flow, and promotes angiogenesis through its vascular endothelial factors, ${ }^{23,24}$ thereby helping to remodel tissue and preventing early leakage. Another factor could be that the pedicled omentum helps to seal the microscopic leakage and shield the juncture crevice against the spread of leaking gastric fluid if cervical anastomotic leakage had occurred. ${ }^{25}$ In our surgery, the right gastroepiploic artery was carefully preserved and the omentum was freed from the transverse colon and divided at the edge adherent to the colon, so that the length of the pedicled omentum and the arterial communications were preserved more fully. Besides, two or three branches of the right gastroepiploic artery were preserved on the pedicled omental flap, which can provide an adequate blood supply.

In the univariate and the multivariate analyses, the statistically significant risk factors associated with esophageal leakage appear to be disease processes that compromise perfusion of the anastomosis. Risk factors identified in the logistic regression analysis suggestive of higher leakage rates include not performing omentoplasty, renal insufficiency, and diabetes. Based on these findings, the use of omentoplasty was also an independent predictor of lower leakage incidence. Interestingly, the typical compromise perfusion of cardiac insufficiency and hypertension were not a significant variable contributing to the end point.

The major limitation of this study is that it is a retrospective study that included a small number of patients at a single institution. However, to our knowledge, it is the first study analyzing the influence of omentoplasty on cervical esophagogastric anastomotic leakage rates after MIE.

\section{Conclusion}

Omentoplasty may prevent anastomotic leakage and decrease leakage-associated mortality in cervical esophagogastrostomy following MIE, which helps to seal microscopic leakage and shield the cervicothoracic juncture crevice against spreading gastric fluid when cervical anastomotic leakage occurs. Further studies are required to fully appreciate the full effect of omentoplasty on leakage rate and its severity before advocating its routine use in clinical practice.

\section{Acknowledgment}

The study was supported by funding from the National Natural Science Foundation of China for J-GD (81472188) and HZ (81702247), the Youth Development Project of Third Military Medical University for HZ (2016XPY17), and the clinical funding from the Xinqiao Hospital of the Third Military Medical University (2016YLC30 and 2016YLC29).

\section{Author contributions}

LJ and J-GD contributed substantially to conception and design and gave final approval of the version to be published. HZ and XL contributed to the analysis and interpretation of all data and drafted the article. DZ, X-FD, and Q-XL revised the article critically for important intellectual content. All authors contributed toward data analysis, drafting and critically revising the paper and agree to be accountable for all aspects of the work.

\section{Disclosure}

The authors report no conflicts of interest in this work.

\section{References}

1. Chen W, Zheng R, Baade PD, et al. Cancer statistics in China, 2015. CA Cancer J Clin. 2016;66:115-132.

2. Pennathur A, Luketich JD. Resection for esophageal cancer: strategies for optimal management. Ann Thorac Surg. 2008;85(2):S751-S756.

3. Rutegard M, Lagergren P, Rouvelas I, Lagergren J. Intrathoracic anastomotic leakage and mortality after esophageal cancer resection: a population-based study. Ann Surg Oncol. 2012;19(1):99-103.

4. DePaula AL, Hashiba K, Ferreira EA, de Paula RA, Grecco E. Laparoscopic transhiatal esophagectomy with esophagogastroplasty. Surg Laparosc Endosc. 1995;5(1):1-5.

5. Cuschieri A, Shimi S, Banting S. Endoscopic oesophagectomy through a right thoracoscopic approach. J R Coll Surg Edinb. 1992;37(1):7-11.

6. Zhou C, Ma G, Li X, et al. Is minimally invasive esophagectomy effective for preventing anastomotic leakages after esophagectomy for cancer? A systematic review and meta-analysis. World J Surg Oncol. 2015;13:269.

7. Bhat MA, Dar MA, Lone GN, Dar AM. Use of pedicled omentum in esophagogastric anastomosis for prevention of anastomotic leak. Ann Thorac Surg. 2006;82(5):1857-1862.

8. Dai JG, Zhang ZY, Min JX, Huang XB, Wang JS. Wrapping of the omental pedicle flap around esophagogastric anastomosis after esophagectomy for esophageal cancer. Surgery. 2011;149(3):404-410.

9. Yuan Y, Hu Y, Xie TP, Zhao YF. Omentoplasty for preventing anastomotic leaks after esophagogastrostomy. Surgery. 2011;149:853-854.

10. Takeuchi $\mathrm{H}$, Miyata $\mathrm{H}$, Gotoh M, et al. A risk model for esophagectomy using data of 5354 patients included in a Japanese nationwide web-based database. Ann Surg. 2014;260(2):259-266.

11. Van Workum F, van der Maas J, van den Wildenberg FJ, et al. Improved functional results after minimally invasive esophagectomy: intrathoracic versus cervical anastomosis. Ann Thorac Surg. 2017;103(1):267-273.

12. Takemura M, Yoshida K, Fujiwara Y. Modified triangulating stapling technique for esophagogastrostomy after esophagectomy for esophageal cancer. Surg Endosc. 2013;27(4):1249-1253.

13. Yuan Y, Wang KN, Chen LQ. Esophageal anastomosis. Dis Esophagus. 2015;28(2):127-137.

14. Sepesi B, Swisher SG, Walsh GL, et al. Omental reinforcement of the thoracic esophagogastric anastomosis: an analysis of leak and reintervention rates in patients undergoing planned and salvage esophagectomy. J Thorac Cardiovasc Surg. 2012;144(5):1146-1150.

15. Sun HB, Li Y, Liu XB, et al. Embedded three-layer esophagogastric anastomosis reduces morbidity and improves short-term outcomes after esophagectomy for cancer. Ann Thorac Surg. 2016;101(3):1131-1138.

16. Liu QX, Deng XF, Wang JS, et al. Use of oesophageal flap valvuloplasty and wrapping suturing technique in preventing postoperative complications after oesophagectomy for oesophageal cancer. Eur J Surg Oncol. 2014;40(10):1355-1360.

17. Urschel JD, Miller JD, Young JE. Omentoplasty reinforcement of cervical esophagogastrostomy anastomoses. Hepatogastroenterology. 2002;49:1. 
18. Ohwada S, Ogawa T, Kawate S, et al. Omentoplasty for cervical esophagogastrostomy following radical esophagectomy with three-field dissection. Hepatogastroenterology. 2000;47(35):1305-1309.

19. Ohwada S, Ogawa T, Kawate S, et al. Omentoplasty versus no omentoplasty for cervical esophagogastrostomy following radical esophagectomy. Hepatogastroenterology. 2002;49(43):181-184.

20. Yoshida N, Baba Y, Watanabe M, et al. Triangulating stapling technique covered with the pedicled omental flap for esophagogastric anastomosis: a safe anastomosis with fewer complications. J Am Coll Surg. 2015;220:e13-e16.

21. Liu QX, Deng XF, Hou B, Min JX, Dai JG. Preventing and localizing esophagogastric anastomosis leakage by sleeve-wrapping of the pedicled omentum. World J Gastroenterol. 2014;20(43):16282-16286.
22. Zheng QF, Wang JJ, Ying MG, Liu SY. Omentoplasty in preventing anastomotic leakage of oesophagogastrostomy following radical oesophagectomy with three-field lymphadenectomy. Eur J Cardiothorac Surg. 2013;43(2):274-278.

23. Konturek SJ, Brzozowski T, Majka I, Pawlik W, Stachura J. Omentum and basic fibroblast growth factor in healing of chronic gastric ulcerations in rats. Dig Dis Sci. 1994;39(5):1064-1071.

24. Ishikawa M, Fjii M, Iuchi M, Miyauchi T, Tashiro S. Effect of intrahepatic omental implantation on angiogenesis in rat liver with hepatic artery ligation. Clin Exp Med. 2001;1(1):27-33.

25. Pap-Szekeres J, Cserni G, Furka I, et al. Transplantation and microsurgical anastomosis of free omental grafts: experimental animal model of a new operative technique in dogs. Microsurgery. 2003;23(5):414-418.
Cancer Management and Research

\section{Publish your work in this journal}

Cancer Management and Research is an international, peer-reviewed open access journal focusing on cancer research and the optimal use of preventative and integrated treatment interventions to achieve improved outcomes, enhanced survival and quality of life for the cancer patient The manuscript management system is completely online and includes

\section{Dovepress}

a very quick and fair peer-review system, which is all easy to use. Visit http://www.dovepress.com/testimonials.php to read real quotes from published authors. 